\title{
Solitary fibrous tumor of the pleura: A rare mesenchymal tu- mor presented with hypoglycemia
}

\author{
Yasser Ali Kamal ${ }^{\mathrm{a}, *}$ \\ ${ }^{a}$ Department of Cardiothoracic Surgery, Faculty of Medicine, Minia University, El-Minya, Egypt.
}

\begin{abstract}
Solitary fibrous tumor (SFT) is a relatively rare primary tumor of the pleura, originating from the mesenchymal cells. The tumor has a non-specific clinical, laboratory, and radiological features. Patients may be asymptomatic or have non-specific clinical features including chest pain, dyspnea, cough, and paraneoplastic symptoms. Complete surgical resection is indicated in all cases to avoid recurrence of the tumor. We described a 49-yearold woman who presented with unexplained right chest pain and repeated attacks of hypoglycemia, associated with pleural SFT.

Keywords: Pleura; thoracic oncology; solitary fibrous tumor; paraneoplastic hypoglycemia; Doege-Potter syndrome
\end{abstract}

\section{INTRODUCTION}

The primary tumors of the pleura are less common than secondary metastatic ones. Malignant mesothelioma is the most common primary tumor; however, other rare pleural tumors exist including solitary fibrous tumor (SFT), lipoma, lymphoma, sarcoma, Askin tumor, extra-skeletal osteosarcoma, and histiocytoma ${ }^{[1]}$.

SFT of the pleura is a relatively rare mesenchymal tumor that had earlier names of localized mesothelioma and pleural fibroma as it was thought to have a mesothelial origin. Most of the cases of SFT occur within the pleura, but it can arise from other sites including subcutaneous tissues, orbit, salivary glands, thyroid gland, nasal cavity, nasopharynx, lung, pericardium, adrenal glands, liver, GI tract, urinary bladder, and prostate ${ }^{[2,3]}$. Here, we describe a case of SFT of the pleura with mild right chest pain and unexplained hypoglycemia.

\section{CASE REPORT}

* Corresponding author: Yasser Ali Kamal Mailing address: Department of Cardiothoracic Surgery, Faculty of Medicine, Minia University, El-Minia, 61111, Egypt.

E-mail: yaser_ali_kamal@yahoo.com, Yasser.Ali@mu.edu.eg Received: 09 December 2020 / Accepted: 23 February 2021
A 49-year-old previously healthy woman presented to our clinic with a 3-month history of mild right chest pain and repeated attacks of confusion and drowsiness improved after eating. There was laboratory evidence of hypoglycemia during the last attack (blood glucose level of $40 \mathrm{mg} / \mathrm{dl}$ ). Chest X-ray showed an opaque shadow on the lower zone of the right side of the chest (Figure 1). The past medical history was unremarkable with normal laboratory investigations. A computed tomography (CT) scan revealed a well-defined right pleural-based soft tissue mass with a long axis diameter of $10 \mathrm{~cm}$ (Figure 2). CT-guided core needle biopsy revealed a spindle cell tumor. The patient underwent the right posterolateral thoracotomy. During surgery, the tumor was located in the right inferior hemithorax within the fifth and sixth intercostal spaces and it was attached to the parietal pleura by a fibrous pedicle. The tumor was completely resected. Grossly, the resected mass was round firm, well circumscribed encapsulated of $4.5 \times 3 \mathrm{~cm}$ in diameter and the cut surface with grayish, yellow and mucoid (Figure 3). Microscopically, there was a bland spindle cell proliferation distributed in a patternless fashion separated by dense collagen stroma, with low mitotic figures less than 3/10 HPFs (Figure 4). On immunohistochemical examination, the tumor was positive for cluster of differentiation (CD34), CD99, and Vimentin and negative for S-100. The 


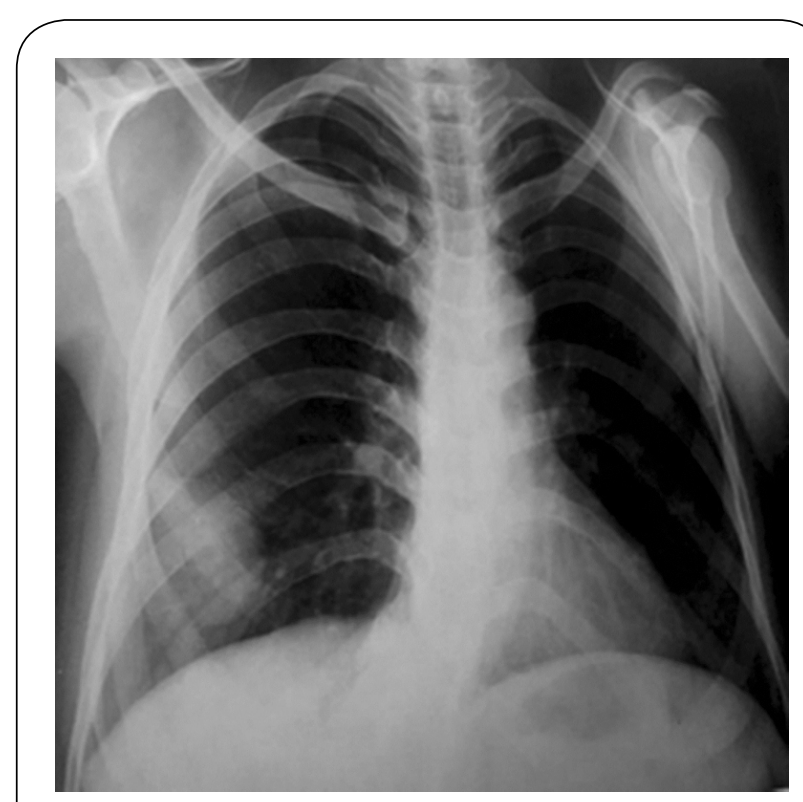

Figure 1. Postero-anterior view of chest X-ray shows an opaque shadow in the lower zone of the right hemi-thorax.

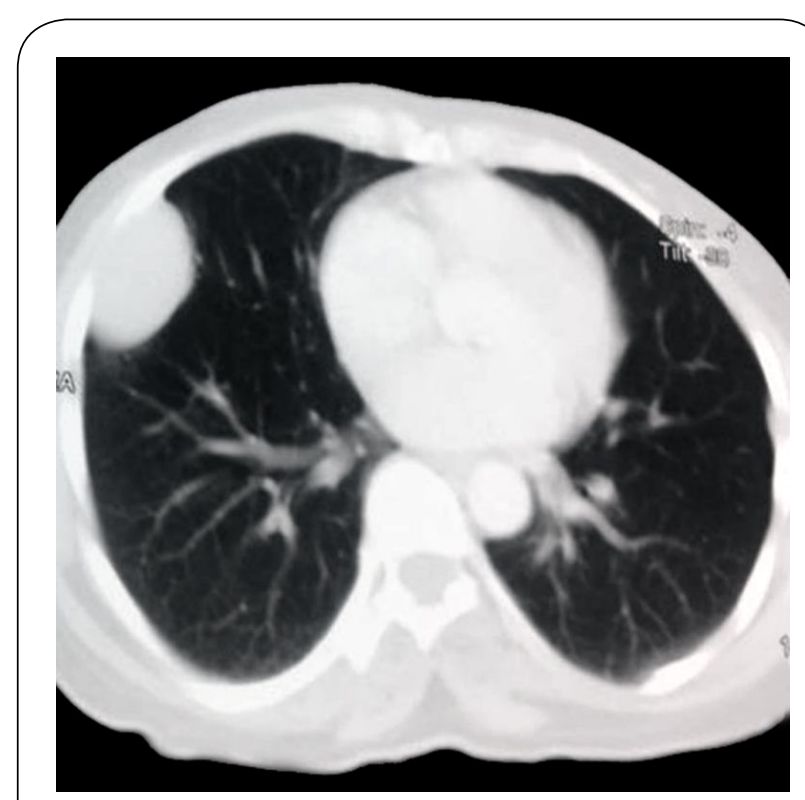

Figure 2. Axial view of chest CT scan shows a right pleuralbased mass.

final diagnosis was benign pleural SFT. The postoperative course was uneventful, with freedom from symptoms and tumor recurrence within two years of followup after surgery.

\section{DISCUSSION}

SFT is a mesenchymal cell tumor most commonly occurs in middle-aged adults, with an equal gender distribution ${ }^{[4]}$. The thoracic SFT occurs equally on the right and left sides and arises from the pleura in most cases, followed by lung, anterior mediastinum, and diaphragm ${ }^{[5]}$. The etiology of SFT remains unclear with the absence of association to smoking or asbestos exposure ${ }^{[6]}$. However, there is a genetic evidence recognizing SFT as a translocation-associated neoplasm, with a consistent association with NAB2-STAT6 gene fusions arising from recurrent intrachromosomal rearrangements on chromosome $12 \mathrm{q}^{[7]}$.

Clinically, SFT presented as a slowly growing painless mass that may produce symptoms due to its large size or pressure effects on the adjacent structures. More than $50 \%$ of cases with SFT are asymptomatic and detected incidentally on chest radiographs or CT. Symptomatic SFT presented with non-specific complaints such as pleuritic chest pain, cough, and shortness of breath ${ }^{[8]}$. The CT features are often quite suggestive but not pathognomonic. On CT scan with contrast, SFT appears as a heterogeneous tumor with acute angles at pleural interface for large masses and obtuse angles for smaller tumors ${ }^{[9]}$. A biopsy is recommended before starting treatment of SFT through open incisional biopsy or by a core needle with a preference for radiological guided biopsy if it is applicable.

Histologically, SFT is a benign tumor although malignant transformation occurs in $20 \%$ of patients ${ }^{[10]}$. Also, SFT has a wide differential diagnosis on the histological examination due to the similarity in cellular architecture and vascular pattern with other tumors such as schwannoma, spindle cell/pleomorphic lipomas, gastrointestinal stromal tumors, and liposarcoma [7]. Immunohistochemistry is useful for the diagnosis and differentiation of SFT from other spindle cell mesenchymal tumors. The most important and valuable immunohistochemical positive markers in SFT are CD34, CD99, Vimentin, Bcl-2 and STAT-6. Additionally, some markers are negative in cases of SFT according to the tumor location, such as $\mathrm{S100}$, smooth muscle actin (SMA), desmin, and calretinin ${ }^{[11]}$.

Surgery is the gold standard treatment for all types of SFT, as its benefit outweigh the risks and the prognosis for malignant SFT is favorable with surgical resection ${ }^{[10]}$. Chemotherapy and consecutive selective embolization of the feeding arteries are alternative options when the SFT is non-resectable or metastatic ${ }^{[12]}$. The goal of the surgical management of SFT relies on wide resection margins and preservation of the surrounding structures. Complete resection with adequate negative margins is essential to reduce the rates of local recurrence and progression to metastatic disease ${ }^{[13,14]}$. SFT is usually cured after complete surgical resec- 


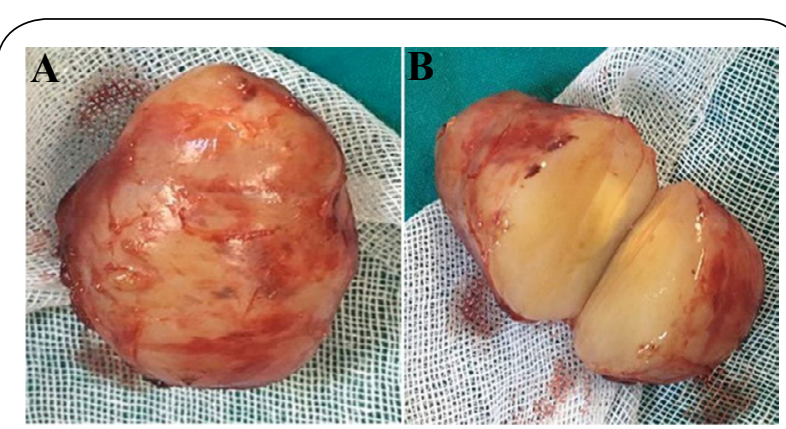

Figure 3. Gross appearance of the surgically excised wellcircumscribed encapsulated tumor (A), with grayish yellow cut surface (B).

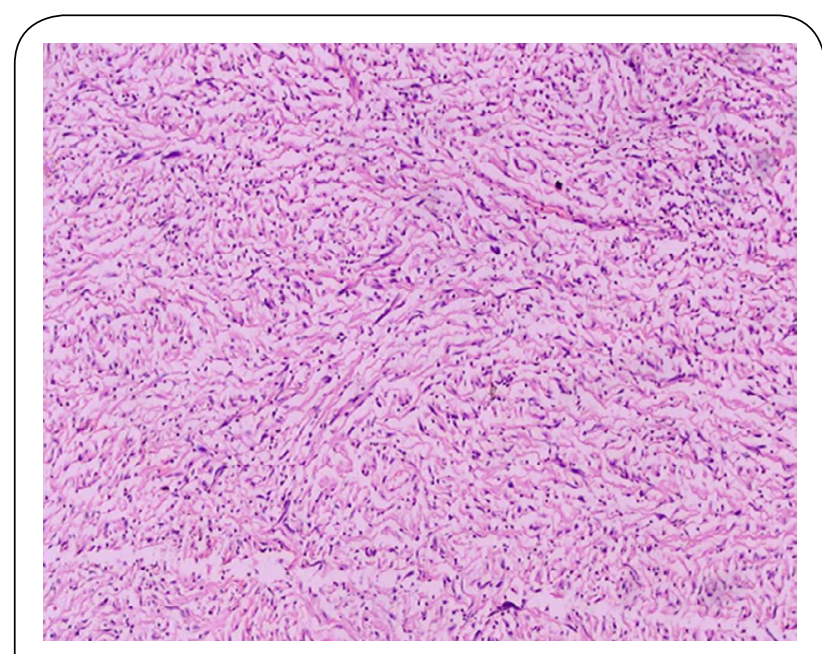

Figure 4. Histopathological image shows spindle-shaped cells with patternless pattern arranged on a collagenous stroma $(\mathrm{H} \& \mathrm{E} \times 100)$.

tion; however, a recent systematic review found that SFTs recurred after 5 years of surgery in at least 23\% of cases and the authors of the review recommended a longer follow up schedule ${ }^{[15]}$. Moreover, the local recurrence has been reported in about $10 \%$ of benign SFT ${ }^{[16]}$ and in more than $60 \%$ of malignant SFTs, which indicates surgical re-resection in cases with positive surgical margins ${ }^{[17]}$. The possibility of repeated recurrence after surgery indicates extended surgical resection if the pleural SFT invades the chest wall, lung, diaphragm, or pericardium ${ }^{[18]}$.

Our case of pleural SFT presented with paraneoplastic hypoglycemia, which is known as Doege-Potter syndrome (DPS). This rare entity occurs due to inappropriate secretion of a high molecular-weight form of insulin-like growth factor type II (IGF-II). This big IGF-II activates insulin receptors, inhibits hepatic gluconeogenesis, and increases glucose uptake with subsequent hypoglycemia ${ }^{[12]}$. Thus, DPS is characterized by non-islet cell hypoinsulinemic hypoglycemia, which may be life-threatening when food intake decreased ${ }^{[19]}$. It is important to consider DPS in a patient with SFT and hypoglycemia, especially when the tumor size is larger than $8 \mathrm{~cm}$ which is thought to be a risk factor for this syndrome ${ }^{[20]}$. The diagnosis of DPS is based on the clinical presentation of hypoglycaemic episodes with SFT in absence of alternative causes or on laboratory findings of increased IGF-II to IGF-I ratio and hypoinsulinemia during attacks of hypoglycemia ${ }^{[21]}$. Treatment of DPS associated with SFT should initially aim to normalize the blood glucose level by high-glucose diet and continuous intravenous infusion of $50 \%$ glucose (up to $50 \mathrm{~g} / \mathrm{hr}$ ), followed by tumor size reduction through surgical resection, chemotherapy, radiotherapy, brachytherapy, and photodynamic therapy. However, complete surgical resection of SFT is the curative therapy for DPS ${ }^{[22]}$.

\section{CONCLUSION}

SFT is a relatively rare soft tissue tumor that often originates from the mesenchymal cells of the pleura. The diagnosis of SFT is difficult due to the absence of symptoms in most of the cases, in addition to nonspecific clinical, laboratory, and radiological features. Some cases may present with paraneoplastic hypoglycemia, known as Doege-Potter syndrome. Surgical resection is the mainstay in the treatment of SFT with or without hypoglycemia. Complete surgical resection is essential to avoid the recurrence of both benign and malignant tumors.

\section{DECLARATIONS}

\section{Authors' contributions}

The author contributed solely to the article.

\section{Conflict of interest}

The author declared that there are no conflicts of interest.

\section{Ethical approval}

Not applicable.

\section{Consent for publication}

The consent was obtained from the patient.

\section{REFERENCES}

1. Sureka, B., Thukral, B. B., Mittal, M. K., Mittal, A., \& Sinha, M. (2013). Radiological review of pleural tumors. The Indian journal of radiology \& imaging, 23(4), 313.

2. Brunnemann, R. B., Ro, J. Y., Ordonez, N. G., Mooney, J., El- 
Naggar, A. K., \& Ayala, A. G. (1999). Extrapleural solitary fibrous tumor: a clinicopathologic study of 24 cases. Modern pathology: an official journal of the United States and Canadian Academy of Pathology, Inc, 12(11), 10341042.

3. Agarwal, V. K., Plotkin, B. E., Dumani, D., French, S. W., Becker, R., \& Lee, P. (2009). Solitary fibrous tumor of pleura: a case report and review of clinical, radiographic and histologic findings. Journal of radiology case reports, 3(5), 16.

4. Demicco, E. G., Park, M. S., Araujo, D. M., Fox, P. S., Bassett, R. L., Pollock, R. E., ... \& Wang, W. L. (2012). Solitary fibrous tumor: a clinicopathological study of 110 cases and proposed risk assessment model. Modern Pathology, 25(9), 1298-1306.

5. Gold, J. S., Antonescu, C. R., Hajdu, C., Ferrone, C. R., Hussain, M., Lewis, J. J., ... \& Coit, D. G. (2002). Clinicopathologic correlates of solitary fibrous tumors. Cancer, 94(4), 1057-1068.

6. Davanzo, B., Emerson, R. E., Lisy, M., Koniaris, L. G., \& Kays, J. K. (2018). Solitary fibrous tumor. Translational gastroenterology and hepatology, 3, 94.

7. Thway, K., Ng, W., Noujaim, J., Jones, R. L., \& Fisher, C. (2016). The current status of solitary fibrous tumor: diagnostic features, variants, and genetics. International Journal of Surgical Pathology, 24(4), 281-292.

8. Hohenforst-Schmidt, W., Grapatsas, K., Dahm, M., Zarogoulidis, P., Leivaditis, V., Kotoulas, C., ... \& Kosan, B. (2017). Solitary fibrous tumor: A center's experience and an overview of the symptomatology, the diagnostic and therapeutic procedures of this rare tumor. Respiratory medicine case reports, 21, 99-104.

9. Salahudeen, H. M., Hoey, E. T. D., Robertson, R. J., \& Darby, M. J. (2009). CT appearances of pleural tumours. Clinical radiology, 64(9), 918-930.

10. Supakul, R., Sodhi, A., Tamashiro, C. Y., Azmi, S. S., \& Kadaria, D. (2015). Solitary fibrous tumor of the pleura: A Rare cause of pleural mass. The American journal of case reports, 16,854 .

11. Geramizadeh, B., Marzban, M., \& Churg, A. (2016). Role of immunohistochemistry in the diagnosis of solitary fibrous tumor, a review. Iranian journal of pathology, 11(3), 195.

12. Han, G., Zhang, Z., Shen, X., Wang, K., Zhao, Y., He, J., ... \&
Liu, X. (2017). Doege-Potter syndrome: a review of the literature including a new case report. Medicine, 96(27), e7417.

13. Sung, S. H., Chang, J. W., Kim, J., Lee, K. S., Han, J., \& Park, S. I. (2005). Solitary fibrous tumors of the pleura: surgical outcome and clinical course. The Annals of thoracic surgery, 79(1), 303-307.

14. Kayani, B., Sharma, A., Sewell, M. D., Platinum, J., Olivier, A., Briggs, T. W., \& Eastwood, D. M. (2018). A review of the surgical management of extrathoracic solitary fibrous tumors. American journal of clinical oncology, 41(7), 687694.

15. Mercer, R. M., Wigston, C., Banka, R., Cardillo, G., Benamore, R., Nicholson, A. G., ... \& Rahman, N. M. (2020). Management of solitary fibrous tumours of the pleura: a systematic review and meta-analysis. ERJ open research, 6(3), 0005-2020.

16. Harrison-Phipps, K. M., Nichols, F. C., Schleck, C. D., Deschamps, C., Cassivi, S. D., Schipper, P. H., ... \& Pairolero, P. C. (2009). Solitary fibrous tumors of the pleura: results of surgical treatment and long-term prognosis. The Journal of thoracic and cardiovascular surgery, 138(1), 19-25.

17. Robinson, L. A. (2006). Solitary fibrous tumor of the pleura. Cancer control, 13(4), 264-269.

18. Forster, C., Roumy, A., \& Gonzalez, M. (2019). Solitary fibrous tumor of the pleura with Doege-Potter syndrome: Second recurrence in a 93-year-old female. SAGE open medical case reports, 7, 2050313X18823468.

19. Schutt, R. C., Gordon, T. A., Bhabhra, R., Cathro, H. P., Cook, S. L., McCartney, C. R., \& Weiss, G. R. (2013). Doege-Potter syndrome presenting with hypoinsulinemic hypoglycemia in a patient with a malignant extrapleural solitary fibrous tumor: a case report. Journal of medical case reports, 7(1), 1-7.

20. Zafar, H., Takimoto, C. H., \& Weiss, G. (2003). DoegePotter syndrome. Medical Oncology, 20(4), 403-407.

21. Ahluwalia, N., Attia, R., Green, A., Cane, P., \& Routledge, T. (2015). Doege-Potter syndrome. The Annals of The Royal College of Surgeons of England, 97(7), e105-e107.

22. Jang, J. G., Chung, J. H., Hong, K. S., Ahn, J. H., Lee, J. Y., Jo, J. H., ... \& Lee, J. K. (2015). A case of solitary fibrous pleura tumor associated with severe hypoglycemia: doegepotter syndrome. Tuberculosis and respiratory diseases, $78(2), 120$. 